\title{
Visual alignment of arteries in coronary cine-angiogram using global motion stabilisation approach
}

\author{
K.A.S.H. Kulathilake ${ }^{1}$, L. Ranathunga ${ }^{1}$, G.R. Constantine ${ }^{2}$ and N.A. Abdullah ${ }^{3}$ \\ ${ }^{I}$ Department of Information Technology, Faculty of Information Technology, University of Moratuwa, Moratuwa. \\ ${ }^{2}$ Department of Clinical Medicine, Faculty of Medicine, University of Colombo, PO. Box 271, No. 25, Kynsey Road, Colombo 08. \\ ${ }^{3}$ Department of Computer System and Technology, Faculty of Computer Science and Information Technology, University of Malaya, 50603 \\ Kuala Lumpur, Malaysia.
}

Revised: 23 May 2016; Accepted: 18 August 2016

\begin{abstract}
Coronary cine-angiogram (CCA) is an invasive medical image modality, which is used to detect stenosis in coronary arteries (CA). Global motion that occurs due to the heart beat is a major obstruction to visually track correlated arterial flow within CCAs. This study devises a novel method to align the main $\mathrm{CA}$ visualised among the CCA frames using global motion stabilisation. In order to stabilise the global motion artifact, motion vectors are calculated through improved template matching technique, which is introduced in this study to match a given CA template with the CCA frame that contains deformed CA tree. The accuracy of the proposed content alignment method was reported as $97.45 \%$. The content aligned global motion stabilised CCA is produced as the output of this study and those CCAs can be used to further enhance the angiography image modality, to determine the functional significance of CA to assess the severity level of the detected stenosis.
\end{abstract}

Keywords: Coronary angiography, correlation coefficient, motion stabilisation, template matching, vessel alignment, visual correlation.

\section{INTRODUCTION}

Coronary cine-angiogram (CCA) is one of the invasive medical image modalities used to detect luminal obstruction or the degree of stenosis in coronary arteries (CA). The diagnosis based on coronary angiogram is subjective because the results are produced mainly through visual judgments, which are done based on the angiogram images taken under the limited number of views (Wagner et al., 2011; Wong et al., 2012). Therefore, overestimations and underestimations of stenosis frequently occur in clinical judgments due to the lack of objective assessment methods in the angiography technique (Tonino et al., 2010). Hence, it is desirable to conduct an objective assessment of the detected stenosis based on the functional significance of CA as recorded in the CCAs (Sinha Roy et al., 2008; Wong et al., 2012).

CCAs are highly influenced by various types of visual problems such as non-uniform illumination, noise and motion artifacts (Takagi et al., 1999; Khan et al., 2008; Truc et al., 2009; Dehkordi et al., 2011; Suh et al., 2011). Non-uniform illumination and noise degrade the visual clarity of vessel structures in CCA frames (Dehkordi et al., 2011). Additionally, CCAs consist of various types of motion artifacts (Moscheni et al., 1995; Yamamoto et al., 2009). There are three main types of motions in CCAs, namely, the global, radial and local motions. Global and radial motions occur due to the systolic and diastolic movements of the heart. Global motion produces rapid displacements of vessel structures from its initial recorded point, and radial motion makes tiny movements in distal parts of the main CA. Local motion occurs due to the flow of the contrast agent within the CA, and it is the important motion category to determine the functional significance of arterial flow (Moscheni et al., 1995). Figure 1 consists of non-uniformly illuminated four consecutive frames of a CCA and graphically elaborate the artifact produced by both global and local motions of a heart. The catheter engaged area shown in each frame of Figure 1 is marked using a white square for better visualisation of the artifact. As a result of global motion, the placement of this marked region varies in the four consecutive frames named as $a, b, c$ and $d$ of Figure 1. It is noteworthy that, the distribution of contrast agent gradually increases in all four images as a result of the local motion. 


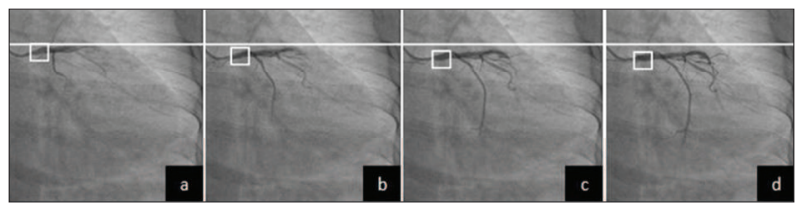

Figure 1: Motion artifacts in CCAs. White square indicates the catheter engaged region

Unlike renal or cerebral angiography, CCAs are implicitly recorded with global motion, thus it changes the placements of vessel structure from one frame to another. Due to this, it is difficult to obtain visual correlation of the vessel structure across the CCA frames sequence (Meijering et al., 1999; Shechter et al., 2006; Kusumawardhani et al., 2011). Nonetheless, the visual correlation of the marked or dyed arteries across the frames is crucial prior to determining the functional significance of the CA. Further, it requires additional computation steps to determine the placement of the vessel's starting point (catheter engage point) in each frame due to the nonexistence of visual correlation. Therefore, it is indeed significant to suggest a method to stabilise global motion artifacts in CCA frames so that the marked arteries can be aligned across the frames based on a selected point in its initial frame.

Although coronary angiography consists of visual problems, global motion artifact and subjective diagnosis, it is still the standard technique for guiding percutaneous coronary intervention in patients with coronary artery disease (Kusumawardhani et al., 2011). Therefore, various research attempts have been undertaken in the recent past to estimate and eliminate the motion artifacts from coronary angiography (Meijering et al., 1999; Yamamoto et al., 2009; Zheng \& Weirong, 2009; Kusumawardhani et al., 2011). Feature descriptor based method, image registration method and optical flow based method are among the major techniques for motion stabilisation in angiograms.

A scale invariant feature transform (SIFT) based motion estimation and video stabilisation technique was described in a recent research study (Kusumawardhani et al., 2011). In this study, SIFT was used to obtain the key points between two consecutive frames. The nearest distance between the SIFT feature in two consecutive frames was calculated during the SIFT feature matching step to determine the global motion vector.

Image registration based approaches for motion estimation and correction have been proposed previously (Meijering et al., 1999). In their proposed method canny edge detection was applied to extract artifact regions. Template matching was applied to compute the displacement vector, and a histogram based method was used as the similarity measure of the template matching. Finally, warping technique was applied to create the motion compensated angiogram images.

Zheng and Weirong (2009) analysed the coronary arterial dynamics using an optical flow based technique and elastic registration. It has been proved that the optical flow based method is physically clear and simple in estimating the object's displacement field from dynamic image sequence. Further, they explained that the elastic registration estimates the displacement field through the globally matching vessel skeletons at different time points.

The proposed motion stabilisation method is implemented based on the template matching technique and it aligns the vessel structures (content align) shown in the visually enhanced frame sequence of the CCA by reducing the global motion artifact. In conventional template matching, the given template is matched with the source image to find the region of interest based on the spatial contents coherence (Bradski \& Kaehler, 2008; Brunelli, 2009). However, when based on angiogram images the conventional template matching will fail because the arteries recorded in angiogram frames are different from frame-to-frame due to vessel structure deformation and motion artifacts. The proposed motion stabilisation method can address this issue successfully and it produces visually aligned CCA frames as the output. Further, the resulting CCA frames are free from global motion artifact and visualise the spreading of contrast agent within the vessel structure starting from one fixed point. Therefore, the resulting CCAs can be later used to formalise a mechanism for the detection and quantification of stenosis regions of CA based on the functional significance of arterial flow.

\section{METHODOLOGY}

Direct CCAs are inputs to the proposed method and the global motion stabilised version of the input CCAs are produced as the output. The Ethics Review Committee of the Faculty of Medicine, University of Colombo has granted the ethical clearance to extract the CCAs, which are required for validating the results of the proposed method. Direct CCAs produced by the Philips Medical System were used for the experiments and were recorded in DCM (digital imaging and communication in medicine) format under the frame rate of $15 \mathrm{fps}$ with 512 $\times 512$ resolution. The proposed method consists of two 
phases, namely, preprocessing and motion stabilisation. The objective of the preprocessing phase is to enhance the input CCA by eliminating the visual problems such as noise, non-uniform illumination and increasing the contrast of the vessels through normalisation. This enhanced CCA frames are fed as input to the motion stabilisation phase. The main objective of the motion stabilisation phase is to align the vessel structures visualised in individual frames by removing the global motion artifact (content alignment). The flow chart of the proposed method is depicted in Figure 2 for further clarifications.

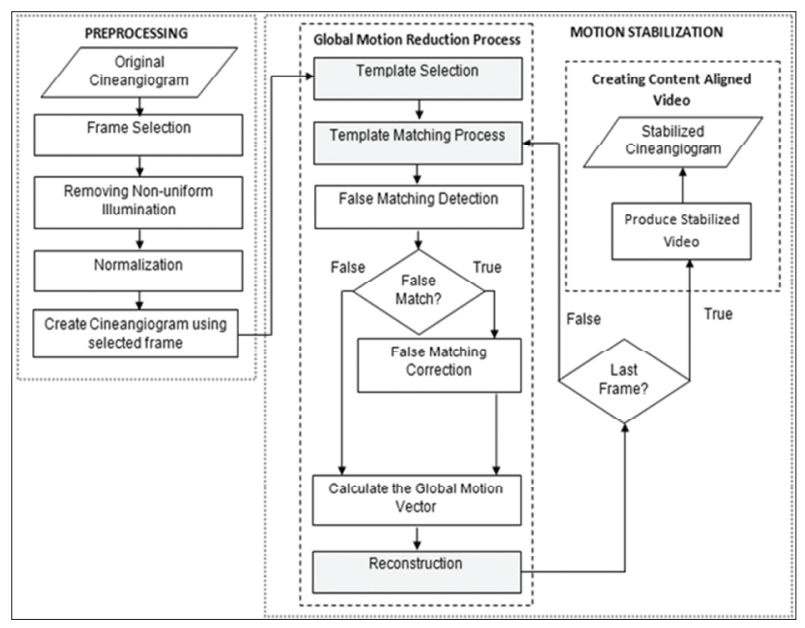

Figure 2: Flow chart of the proposed global motion stabilisation method

\section{Preprocessing phase}

\section{Frame selection}

Within this step, the CCA frames which visualise the filling of the contrast agent within the vessel structure were extracted directly from the CCA. The selected frames are important because they demonstrate the functional significance of CA. Once selected, the frames were repeatedly processed to remove some identified visual problems such as noise, non-uniform illumination and low contrast.

\section{Removal of noise and non-uniform illumination}

It was revealed that the CCA frames consist of salt-andpepper noise and non-uniform illumination as major visual degradations, which lead to some incorrect results in subsequent matching procedures (Dehkordi et al.,
2011; Kusumawardhani et al., 2011). In order to reduce salt-and-pepper noise, a median filter with kernel size $3 \times 3$ was applied to the selected CCA frames. Subsequently, a homomorphic Butterworth low pass filter was applied to extract the illumination component from those selected CCA frames to obtain the illumination uniformity of the CCA frames.

\section{Normalisation}

The processed frames were then normalised to obtain the contrast among the vessel structures. Equation (1) represents the normalisation technique applied in the preprocessing phase.

$$
N(i, j)= \begin{cases}M_{d}+\sqrt{\frac{V_{d}(i m g(i, j)-M)^{2}}{V}} \text { if } \operatorname{img}(i, j)>M \\ M_{d}-\sqrt{\frac{V_{d}(i m g(i, j)-M)^{2}}{V}} \quad \text { otherwise }\end{cases}
$$

where $M$ and $V$ denote the estimated mean and variance of the input image and $M_{d}$ and $V_{d}$ are desired mean and variance values, respectively. $N(i, j)$ is the output image and $\operatorname{img}(i, j)$ denotes the input image where $i$ and $j$ represent the pixel position of both input and output images. According to the experimental results $M_{d}$ was set as $M / 2$ and $V_{d}$ was set as $(V \times 4)$ to obtain better results. Radial distance and order parameters of Butterworth low pass filter and $M_{d}, V_{d}$ parameters of normalisation are adjustable according to experimental setups. Figure 3 depicts the original CCA frame and the respective preprocessed CCA frame for further clarification.

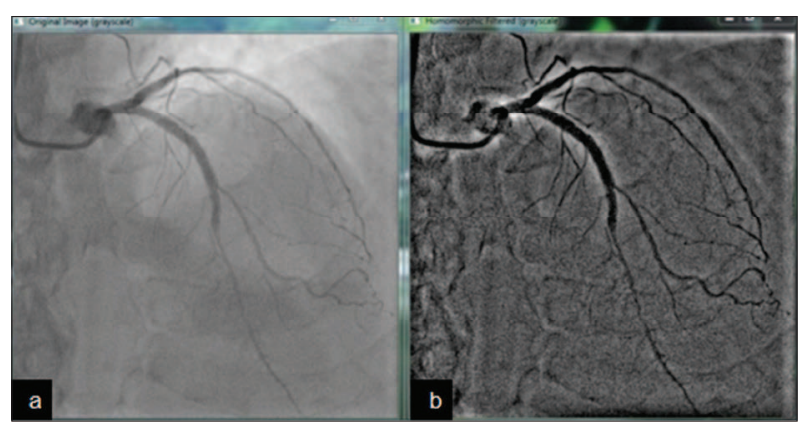

Figure 3: Preprocessing step: (a) frame extracted from direct CCA; (b) corresponding preprocessed frame of (a) 


\section{Motion stabilisation phase}

The motion stabilisation phase contains two main sub processes, namely, global motion reduction process and content aligned video creation process. The global motion reduction process is an iterative process and within the iteration, new global motion stabilised frames are generated from the input preprocessed video frames. Later, the content aligned global motion stabilised CCA is created within the content aligned video creation process. The main steps under these sub processes of the motion stabilisation phase are further described in the following sections.

\section{Template selection}

The global motion reduction process starts with the template selection step. In this step, the initial template is selected interactively from the first frame of the input preprocessed CCA. As shown in Figure 4(a), the selected template is located at $F_{x, y}$ point of the initial frame and it has $(w)$ width and $(h)$ height. The values of $w$ and $h$ depend on the pixel width and height of the arbitrarily selected template. In addition to the template selection, it is required to select another region around the selected template as the search window, in order to set the boundary for the subsequent template matching step. Figure 4(b) depicts the template search window located at $G_{x, y}$ point of the initial frame. It has $m$ width and $n$ height where $m>w$ and $n>h$. The success of matching steps depends on the selected template's visual contents. Therefore, selecting a template around the catheter engaged area visualised in the first frame of the CCA provides successful results during the template matching steps, because the catheter engaged area is clearly visualised in all recorded frames of most CCA cases. Hence, it is recommended to select the initial template from such an area in the first frame of the CCA to be processed.
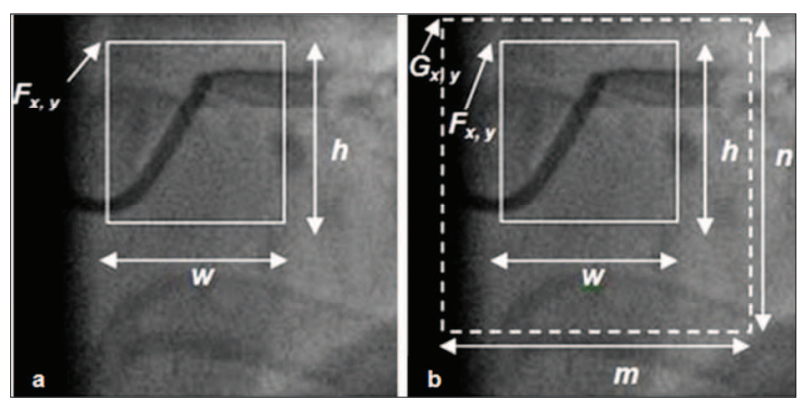

Figure 4: Template selection (a) selected initial template; (b) selected template matching window

\section{Template matching step}

The template matching step begins after the template selection step and it repeats until the last frame of the CCA is processed. Within this step, the template selected from the initial frame (location: $F_{x, y}$, dimensions: $w \times h)$ is matched with the next frame using the search window of $(m)$ width and $(n)$ height. The correlation coefficient (CC) is selected as the similarity measure for the template matching step because it produces the lowest false matching occurrences in the preliminary experiment (Bradski \& Kaehler, 2008; Brunelli, 2009). Matching point is denoted as $C_{x, y}$ where $C_{x, y} \neq F_{x, y}$. Inequality between $C_{x, y}$ point and $F_{x, y}$ point is caused by the global motion, which occurs due to the heart beat. The illustration of the template matching step is depicted in Figure 5.

\section{False matching detection}

There is a very low probability of matching the selected template with the current frame without representing a visual spatial content similarity (Table 2). This type of match is considered as a false positive (FP) match. As a consequence, the matched region contains a similar intensity distribution with the visually mismatched representation, compared to the selected template in the previous frame. Figure 6 depicts this exception clearly. It happens because of the deformations in vessel structures due to the motion artifacts. This exception affects the motion stabilisation defectively.

In the proposed method, a verification technique has been implemented for template matching to detect and correct the FP matches. Further, it has been experimentally observed that it contains larger displacements between $F_{x, y}$ and $C_{x, y}$ points when a FP match occurs. In addition to that, the occurrence of shape mismatches has been observed among the features of matching templates in some FP matching instances. The shape similarity between the template region in the previous frame and the template matched region in the current frame was determined based on the histogram based oriented gradient (HOG) descriptor. Euclidean distance between the HOG descriptor values (HOG distance $-D_{H O G}$ ) obtained for both template region in the previous frame and the template matched region in the current frame was computed. In order to identify the FP templates, HOG distance value was compared with a threshold HOG distance $\left(D_{\text {HOGThreshold }}\right)$ value, which was determined during the preliminary experiment. The following algorithm was implemented to detect the FP matching occurrences. 
FP Detection ( $\left.d_{\text {mean }}, d_{\text {std }}, d_{\text {temp }}\right)$

begin

ifd $_{\text {temp }}==0 \|\left[\left(\mathrm{d}_{\text {mean }}-\mathrm{d}_{\text {std }}\right)<=\mathrm{d}_{\text {temp }}<=\left(\mathrm{d}_{\text {mean }}+\mathrm{d}_{\text {std }}\right)\right]$ then

Correct match

else

$$
\begin{aligned}
& \text { ifd }_{\text {temp }}>\left(\mathrm{d}_{\text {mean }}+\mathrm{d}_{\text {std }}\right) \text { then } \\
& \quad \text { if } \mathrm{d}_{\text {temp }} /\left(\mathrm{d}_{\text {mean }}+\mathrm{d}_{\text {std }}\right)>\text { distanceFactor then }
\end{aligned}
$$

Detect as FP matching

Apply correction process

else

compute $\mathrm{D}_{\mathrm{HOG}}$
if $\left(\mathrm{D}_{\mathrm{HOG}}>=\mathrm{D}_{\text {HOGThreshold }}\right)$ then

Detect as FP match

Apply correction process

else

Correct match

end if

end if

else

if $\left(d_{\text {mean }}-d_{\text {std }}\right) / d_{\text {temp }}>$ distanceFactor then Detect as FP matching

Apply correction process

else

$$
\begin{aligned}
& \text { Compute } \mathrm{D}_{\mathrm{HOG}} \\
& \text { if }\left(\mathrm{D}_{\mathrm{HOG}}>=\mathrm{D}_{\text {HOGThreshold }}\right) \text { then } \\
& \text { Detect as FP match } \\
& \text { Apply correction process }
\end{aligned}
$$

else

Correct match

end if

end if

end if

end if

end

NOTE: FP stands for False Positive. $d_{\text {temp }}, d_{\text {mean }}, d_{\text {std }}$ are the displacement between the templates, mean template displacement and standard deviation of template displacement of the CCA being processed, respectively. Distance factor (distanceFactor) is a constant and it has been empirically set as 2 for this study.

If there is any FP matching occurrence, the re-correction step will begin. The re-correction was done using the matched point obtained in the previous template matching iteration, which is denoted as $C_{x, y}$. For example, if the FP detection occurs in the $n^{\text {th }}$ iteration of the global motion reduction process, it will use the matched coordinates obtained in the $(n-1)^{\text {th }}$ iteration to recorrect it. In this re-correction process, the re-corrected template matching point is marked explicitly from $P_{x, v}$ position in the current frame where $P_{x, y}$ equals the $C_{x, y}^{x, y}$ point of the previous iterations' template matching step.
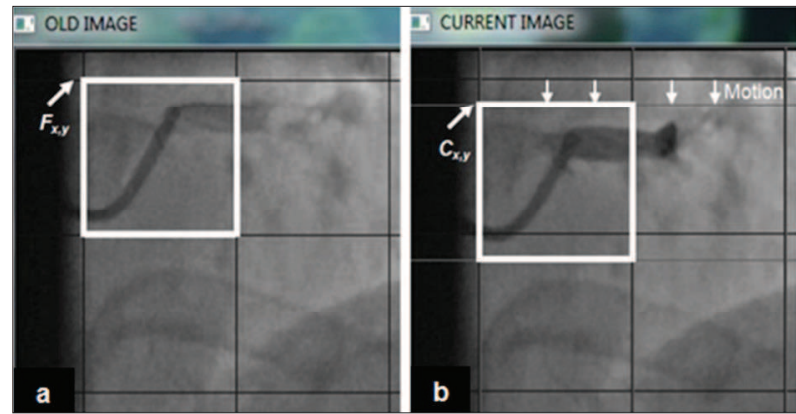

Figure 5: Template matching step between two consecutive frames: (a) selected template at $F_{x, y}$ point; (b) matched template at $C_{x, y}$ point

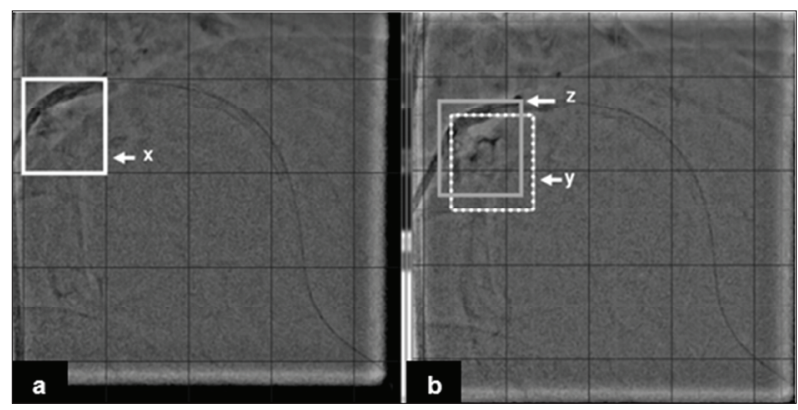

Figure 6: FP template matching occurrence (a) initial frame; $x$ denotes the selected template; (b) matching frame; $y$ denotes the FP template and $z$ denotes the expected TP template 
It was revealed that this re-correction approach does not provide the best matched location for the selected template. Instead, it provides near equal matched area. The reason for that is: the deformation that occurs in vessel structures due to the radial motion among the arteries is visualised in both re-corrected matching region of the current frame and template. Therefore, it is required to smooth the re-corrected point to find the best match point. Euclidean distance between the template and the matched region in the current frame is taken as the metric to find the best matched point.

In order to smoothen the re-corrected point, the Euclidean distance is calculated between the template at $F_{x, y}$ point of the previous frame and the re-corrected template marked in $P_{x, y}$ point in the current frame, and the result is denoted as $E D_{i}$. Subsequently, the Euclidean distance values are calculated separately between the template at $F_{x, y}$ point of the previous frame and the possible matching points, which can be obtained from a region around the $P_{x, y}$ point (block mating). At the end of this block matching, the best matched block to the template at $F_{x, y}$ point of the previous frame is obtained and it is denoted as $E D_{m}$. Finally, the coordinate point which provides $E D_{i}$ is selected as the re-corrected best matched location if $E D_{i}<=E D_{m}$. Otherwise $E D_{m}$ is selected as the re-corrected best matched point during the FP correction step.

\section{Calculating the global motion vector}

In order to construct the global motion stabilised frames, it is required to compute the motion vector $M_{x y}$ of two consecutive frames using the following equation;

$$
M_{x, y}=\left(C_{x}-F_{x}, C_{y}-F_{y}\right)
$$

where $C_{x}$ and $C_{y}$ denote the $x, y$ coordinates of the matched point obtained from $C_{x, y}$ point in the current frame, and $F_{x}$ and $F_{y}$ denote the $x, y$ coordinates of the selected template point at $F_{x, y}$ point in the previous frame.

\section{Construction of motion stabilised frame}

In order to construct a motion stabilised frame, the current frame is translated into a calculated distance towards the direction determined by the motion vector. If $\left(C_{x}-F_{x}\right)$ is negative, the current frame must be shifted to the right direction; else it is shifted to the left. Similarly, if $\left(C_{y}-F_{y}\right)$ is negative, the current frame must be shifted downwards; else it is shifted upwards. As a result of this translation, a new frame is constructed and it is stored within the system to create the content aligned video in the second process of the video stabilisation phase. Further, it is used as the input for the template matching step of the next iteration for selecting the template from $F_{x, y}$ point with dimensions of $(w \times h)$. After the first iteration, the aforementioned template selection step is done automatically using the newly constructed frame produced in the previous frame construction step of the global motion reduction process. This procedure is repeated until the last frame of the input CCA and a set of global motion eliminated angiogram images are produced as the output.

\section{Process of creating content aligned video}

Content aligned video creation process creates a global motion stabilised video using the global motion eliminated frames produced by the global motion reduction process. The frame rate and size of the frames of the motion stabilised video are set as same as the input direct CCAs' frame rate and size to maintain the angiography standards.

\section{RESULTS}

\section{Dataset}

A dataset of 60 CCAs, which belongs to 20 randomly selected patient cases has been selected to validate the proposed method. Each patient case consists of three CCAs, which were recorded under three standard angiography views, namely, left anterior oblique (LAO) cranial, anterior posterior (AP) caudal views of left coronary artery (LCA), and LAO cranial view of right coronary artery (RCA).

\section{Validation method}

Validation process of the proposed content aligned video creation method contains four main steps, namely, finding the best similarity measure for template matching process, finding the value for $D_{\text {HOGThreshold }}$, setting a value for distance factor of FP matching detection, and assessing the robustness of the proposed method.

\section{Finding the best similarity measure for template matching}

In order to find the best similarity measure for template matching, the proposed content aligned video creation method was executed separately under the six different similarity measures defined in the open source computer vision and machine learning software library called OpenCV. Hence, $1372 \times 6$ total template matching 
steps were presented in the executed dataset under each similarity measure. Further, the visually observed template matching judgment, calculated HOG distance $\left(D_{H O G}\right)$ and the Euclidean distance between matched templates for each matching step were recorded.

Each matching instance is determined both as TP or FP matching based on the visually observed template matching judgement made by experts, and is used as

Table 1: TP matching percentage under different similarity measures

\begin{tabular}{lc}
\hline Similarity measure & True positive matching \% \\
\hline Square difference & 83.61 \\
Square difference normalised & 87.66 \\
Correlation & 90.31 \\
Correlation normalised & 86.19 \\
Correlation coefficient & 91.76 \\
Normalised correlation coefficient & 91.72 \\
\hline
\end{tabular}

the ground truth for the validation process. Afterwards, the TP matching percentage for each CCA under the six similarity measures was computed separately to determine the best similarity measure. Based on the results depicted in Table 1, CC was selected as the best similarity measure for the proposed method because it possesses the highest TP matching percentage. The TP matching percentage is calculated using the ratio between the total TP matches and the total template matching steps. Table 2 lists the TP matching percentage obtained for each similarity measure.

Further, Table 2 Test 1 columns summarise the TP matching results obtained under the $\mathrm{CC}$ similarity measure for each test case according to the selected angiography view. Moreover, the analysis results have shown TP matching percentages under the selected views as follows; LAO cranial view of RCA: $91.68 \%$; AP caudal view of LCA: $92.47 \%$; LAO cranial view of LCA: $91.11 \%$. The average TP matching percentage was reported as $91.76 \%$.

Table 2: TP matching results of CCAs under the CC similarity measure ( $D_{\text {HOGThreshold }}=43$ for validation)

\begin{tabular}{|c|c|c|c|c|c|c|c|c|c|}
\hline \multirow[b]{2}{*}{$\begin{array}{l}\text { Test } \\
\text { case }\end{array}$} & \multicolumn{3}{|c|}{$\begin{array}{c}\text { RCA } \\
\text { LAO_CRANIAL }\end{array}$} & \multicolumn{3}{|c|}{$\begin{array}{c}\text { LCA } \\
\text { AP_CAUDAL }\end{array}$} & \multicolumn{3}{|c|}{ LAO_CRANIAL } \\
\hline & $\begin{array}{c}\text { Total } \\
\text { matches }\end{array}$ & $\begin{array}{c}\text { Test } 1 \text { - TP } \\
\text { matches }\end{array}$ & $\begin{array}{l}\text { Test 2- TP } \\
\text { matches }\end{array}$ & $\begin{array}{c}\text { Total } \\
\text { matches }\end{array}$ & $\begin{array}{c}\text { Test } 1 \text { - TP } \\
\text { matches }\end{array}$ & $\begin{array}{c}\text { Test } 2 \text { - TP } \\
\text { matches }\end{array}$ & $\begin{array}{c}\text { Total } \\
\text { matches }\end{array}$ & $\begin{array}{l}\text { Test } 1 \text { - TP } \\
\text { matches }\end{array}$ & $\begin{array}{c}\text { Test } 2 \text { - TP } \\
\text { matches }\end{array}$ \\
\hline 1 & 19 & 19 & 19 & 13 & 13 & 13 & 23 & 23 & 20 \\
\hline 2 & 23 & 23 & 23 & 21 & 6 & 21 & 44 & 44 & 44 \\
\hline 3 & 20 & 20 & 20 & 26 & 26 & 26 & 12 & 12 & 12 \\
\hline 4 & 19 & 19 & 19 & 16 & 16 & 16 & 19 & 19 & 19 \\
\hline 5 & 15 & 15 & 15 & 22 & 22 & 22 & 18 & 18 & 18 \\
\hline 6 & 17 & 17 & 17 & 23 & 23 & 23 & 18 & 18 & 18 \\
\hline 7 & 30 & 30 & 30 & 36 & 36 & 36 & 36 & 36 & 23 \\
\hline 8 & 33 & 33 & 33 & 36 & 36 & 36 & 30 & 30 & 30 \\
\hline 9 & 29 & 29 & 29 & 34 & 34 & 34 & 30 & 30 & 30 \\
\hline 10 & 28 & 28 & 28 & 36 & 36 & 36 & 20 & 20 & 20 \\
\hline 11 & 21 & 21 & 21 & 24 & 8 & 24 & 18 & 18 & 18 \\
\hline 12 & 40 & 26 & 40 & 32 & 32 & 32 & 24 & 24 & 24 \\
\hline 13 & 30 & 30 & 30 & 23 & 23 & 23 & 18 & 2 & 18 \\
\hline 14 & 24 & 24 & 24 & 23 & 23 & 23 & 24 & 18 & 24 \\
\hline 15 & 12 & 12 & 12 & 24 & 24 & 24 & 24 & 11 & 24 \\
\hline 16 & 22 & 22 & 22 & 23 & 23 & 23 & 23 & 18 & 18 \\
\hline 17 & 21 & 7 & 21 & 13 & 13 & 13 & 21 & 21 & 21 \\
\hline 18 & 19 & 9 & 9 & 20 & 20 & 20 & 21 & 21 & 21 \\
\hline 19 & 19 & 19 & 19 & 8 & 8 & 8 & 15 & 15 & 15 \\
\hline 20 & 16 & 16 & 16 & 12 & 8 & 8 & 12 & 12 & 12 \\
\hline Total & 457 & 419 & 447 & 465 & 430 & 461 & 450 & 410 & 429 \\
\hline$\%$ & & 91.68 & 97.82 & & 92.47 & 99.12 & & 91.11 & 95.33 \\
\hline
\end{tabular}




\section{Finding a value for $D_{\text {HOGThreshold }}$}

Initially, the visually observed template matching judgement was compared with the recorded $D_{H O G}$ values for each matching instance of CCAs separately. During this observation, the occurrence of critical shape dissimilarities when $D_{H O G}$ is greater than or equal to 43 has been clearly disclosed. Hence, the value 43 was selected as the $D_{\text {HOGThreshold }}$ distance value and if $D_{H O G} \leq 43$, the matching is considered as a TP match, otherwise it is considered as a FP match. Figure 7 depicts the TP and FP matched templates and HOG descriptor representations for further clarification.

\section{Setting a value for distance factor}

The value for distance factor is decided using the template distance $\left(d_{\text {temp }}\right)$ values obtained for template matching records. In order to determine this, the template distance $\left(d_{\text {temp }}\right)$ values, which are not placed in the matching region and containing the HOG distance $\geq 43$ have been extracted. In other words, it can be justified that those selected records are not placed in the matching region and do not represent the shape similarity. Therefore, it is necessary to calculate the distance ratio for selected upper bound and lower bound records in order to mark the distance margin for determining these total mismatch occurrences. Hence, the distance ratio for upper and lower bounds were calculated as follows;

Upper bound distance ratio $=\mathrm{d}_{\text {temp }} /\left(d_{\text {mean }}+d_{\text {std }}\right)$

Lower bound distance ratio $=\left(d_{\text {mean }}-d_{\text {std }}\right) / d_{\text {temp }}$.

Out of the calculated distance ratios the minimum distance ratio is selected as the distance factor and it is denoted as 2.

\section{Assessing the robustness of the proposed method}

The proposed method was executed for the second time using the same dataset and the same template parameters used in the first test. In this execution the template matching validation algorithm was enabled to detect and correct FP matches. During this execution CC was set as the similarity measure for template matching operation and value 43 was set as the $D_{\text {HOGThreshold }}$. Table 2 Test 2 columns enlist the TP matches obtained as results for this execution. Analysis results of this execution have revealed TP matching percentages under the selected views as follows; LAO cranial view of RCA: $97.82 \%$; AP caudal view of LCA: $99.12 \%$; LAO cranial view of
LCA: $95.33 \%$. The average TP matching percentage was reported as $97.45 \%$.

Figure 8 lists four consecutive frames extracted from a test case to visually compare the results of the proposed content alignment method. It contains the original nonmotion stabilised frames and respective motion stabilised frames constructed during the execution of the proposed method.

\section{DISCUSSION}

In this study, a global motion stabilised CCA creation method and its implementation using a simple image registration technique called template matching has been proposed (Kumar et al., 2007; Bradski \& Kaehler, 2008; Mahalakshmi et al., 2012). Conventional template matching matches the given template with the reference image by checking the spatial resemblance of intensities, and the matching features must not be isotropic and scale invariant. Empirical results of this study have revealed 1259 steps out of 1372 total template matching steps as TP matches, and as a percentage it is $91.76 \%$. Table 2 test 1 results clearly elaborate these figures.
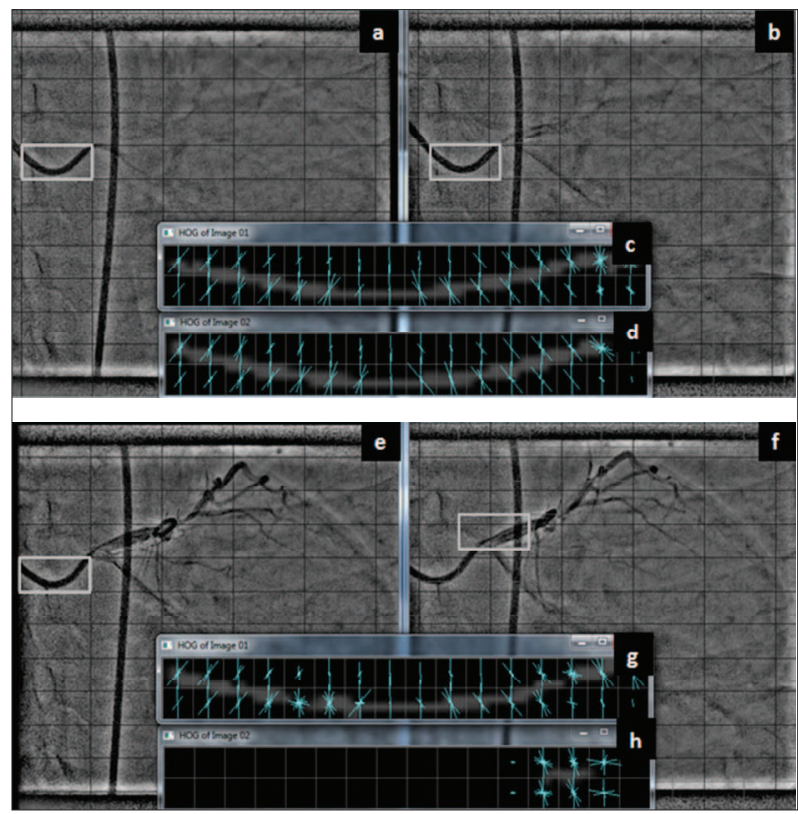

Figure 7: Detection of TP and FP matching occurrence based on HOG descriptor: (a) (b) and (e) (f) consecutive frames with matched templates; (c) (d) HOG descriptors of TP match $\left(D_{H O G}=17.6416\right) ;(\mathrm{g})(\mathrm{h})$ HOG descriptors of FP templates $\left(D_{H O G}=49.4431\right)$ 

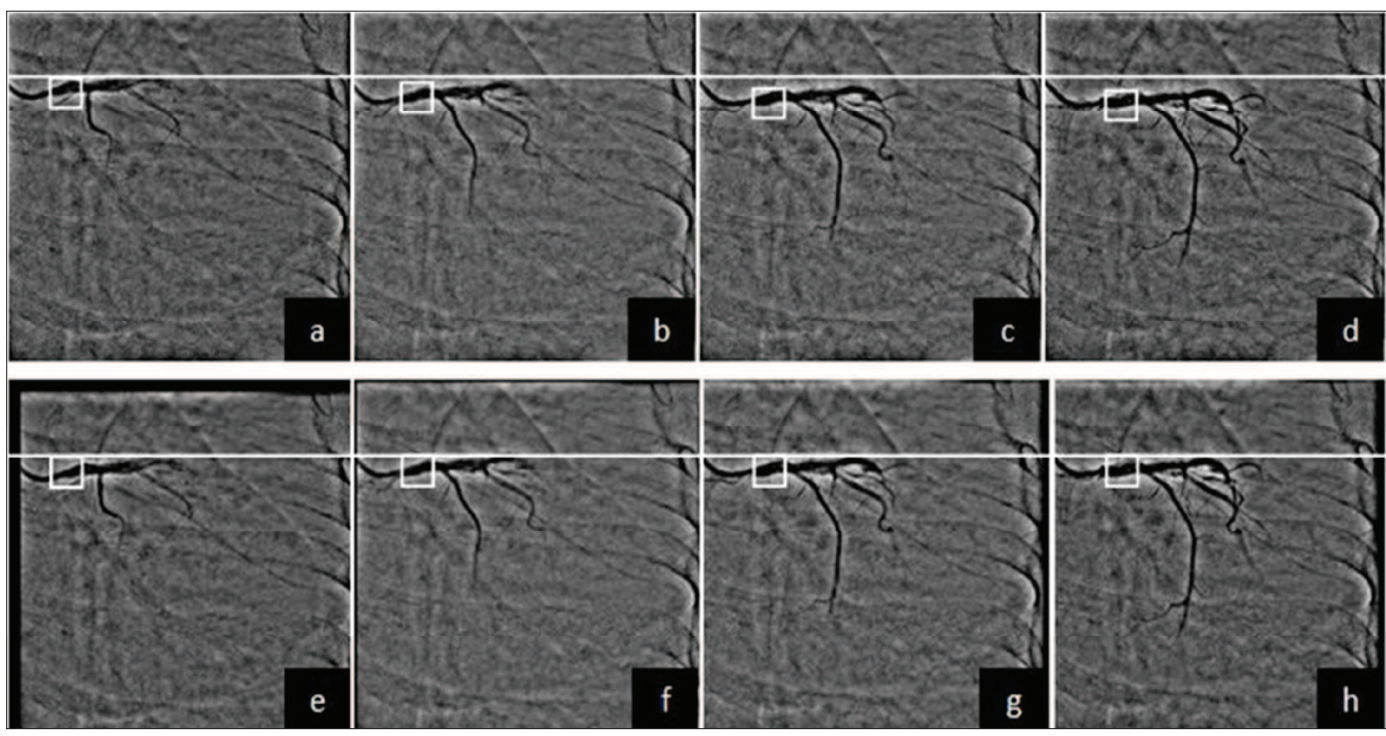

Figure 8: Frames of both original and processed CCA: (a), (b), (c) and (d) represent enhanced frames and (e), (f), (g) and (h) represent the respective content aligned frames

The results further emphasise that the proposed method contains a chance of having FP matching instances $(8.24 \%)$. As a result of these FP matches, incorrectly stabilised CCAs are formed. The main reasons for these FP matches are; the occurrence of radial motion artifacts in vessels and contrast agent concentration variation among the frames. Therefore, rapid spatial differences between the template and the match region in the reference frame can occur. The proposed motion stabilisation method has been implemented with a FP match detection and correction mechanism to improve the TP matching rate further. This improvement is clearly emphasised by the Table 2 test 2 results. According to the results, 1337 steps out of 1372 total template matching steps were reported as TP matches, and as a percentage it is $97.45 \%$. Hence, it improves the conventional template matching accuracy so that it can be adapted for matching the deformed structures represented in CCAs.

An algorithm has been implemented to detect and correct the FP matches in template matching and it was the major improvement suggested to advance the results provided by the conventional template matching technique. According to this proposed algorithm, FP matches are detected based on the displacement of the matched templates and HOG descriptor, because it has been experimentally observed that some FP matches contain rapid distance displacements and some FP matches contain shape dissimilarities between the matched templates. As depicted in Figure 7, HOG descriptor was used to determine the shape dissimilarities and was computed based on the segmented vessel regions depicted in the matched templates.

According to the experimental results, it has been revealed that the proposed adaptive template matching approach provides the matching results by considering both the spatial intensity and feature resemblance among the reference and matched templates. Therefore, unlike the conventional template matching, this novel adaptive template matching method can tolerate the structural deformations in matching an image significantly, and provide visually acceptable matching results. This is the major improvement that has been suggested for the conventional template matching as a result of this research study, so that it can be adaptively matched with the templates from the angiograms with deformed vessel structures.

The global motion stabilisation phase of the proposed method can provide the expected content alignment of the vessel structure (foreground) among the frames. This can be visually judged by looking at the catheter engaged area of both the original and stabilised frame sequences shown in Figure 8. The catheter engaged area is marked as a white square and according to the results, motion stabilised frames depict catheter engaged area in a fixed location. This happens as a result of reconstructing the frame by eliminating the global motion. This frame reconstruction is done based on the calculated motion vector. Hence, it is possible to detect larger motion gaps and eliminate them significantly. As a result of that, 
continuous and smooth contrast agent flow within the vessel structure is visualised starting from the catheter engaged point. This visual correlation obtained among the CCA frames could be used for segmentation of blood vessels among the CCA frame sequence starting from a fixed seed point. Hence, the visual correlation among the CCA frame sequences obtained as a result of this study can be considered as an improvement suggested for the coronary angiography image modality.

It has been reported that the traditional filtering methods slow down the template matching process because the template needs to be matched to every location in the image and the matching involves elementby-element floating point multiplications (Tanimoto, 1981). In order to improve the template matching efficiency, a new template search window of $256 \times 256$ pixel dimension has been suggested instead of using the default template search window defined by the OpenCV vision library. This new template search window has reduced the size of the default template matching window by 2 factors. Hence, it improves the efficiency of the template matching process by reducing the number of comparisons done to filter the matched template point under this modified template search window.

Similar research work has been reported in a recent study and it has described global motion stabilisation of CCAs using scale-invariant feature transform (SIFT) descriptor (Kusumawardhani et al., 2011). According to their study, the global motion vector has been determined based on SIFT feature matching between the two consecutive frames. We have empirically observed that SIFT provides many miss-corresponding key point pairs in angiography. Therefore, finding the corresponding matching features is ambiguous. As a result of that, it provides erroneous global motion vectors for motion compensation.

The proposed method works successfully with the camera motion free CCAs. Due to camera motion, the template area can be shifted out from the captured frame area in some matching instances, and the reconstruction of global motion reduction frame becomes highly difficult in such situations. Hence, it is required to input camera motion free CCAs to this algorithm to obtain successful results.

\section{CONCLUSION}

This study introduces a novel approach to obtain visual alignment of the arterial flow visualised in the CCA frames by removing the global motion artifact.
The proposed method was implemented based on the improved template matching technique that can adapt to match the deformed vessel structures in CCAs. According to the results, it reports $97.45 \%$ matching accuracy and the conventional template matching is $91.76 \%$ in angiography domain. The content aligned CCAs produced from this study would extend research directions to obtain the corelation of vessel segmentation among the CCAs' frames, and consequently determine the functional information of the CA such as blood flow rate and flow velocity for improved diagnosis of heart disease.

\section{Acknowledgement}

This investigation received financial support from the National Science Foundation, Sri Lanka under Grant No. NSF/SCH/2013/06. The authors thank the Cath Lab staff members of the Cardiology Unit, The National Hospital of Sri Lanka for the support given to extract required data for this study.

\section{REFERENCES}

1. Bradski G. \& Kaehler A. (2008). Learning OpenCV: Computer Vision with the OpenCV Library, $1^{\text {st }}$ edition. O'Reilly Media, Sebastopol, USA.

2. Brunelli R. (2009). Template matching techniques in computer vision: theory and practice, pp. $121-122$. John Wiley \& Sons, USA.

DOI: https://doi.org/10.1002/9780470744055

3. Dehkordi M.T., Sadri S. \& Doosthoseini A. (2011). A review of coronary vessel segmentation algorithms. Journal of Medical Signals and Sensors 1(1): $49-54$.

4. Khan M.A.U., Bahadur Khan R., Bilal S., Jamil A. \& Ali Shah M. (2008). Enhancement of angiogram images using pseudo color processing. Information Technology Journal 7(1): $210-214$.

5. Kumar M.S.D., Shen D., Wei L., Turlapthi R. \& Suri J.S. (2007). Motion correction strategies for interventional angiography images: a comparative approach. IEEE International Conference on Image Processing (ICIP 2007), 16 September - 19 October, pp. $497-500$.

DOI: https://doi.org/10.1109/icip.2007.4379000

6. Kusumawardhani A., Mengko T., Fahri I., Soerianata S., Firman D. \& Zakaria H. (2011). Coronary angiogram stabilisation for QuBE values calculation using SIFT method. $2^{\text {nd }}$ International Conference on Instrumentation, Communications, Information Technology, and Biomedical Engineering (ICICI-BME), pp. $111-116$. DOI: https://doi.org/10.1109/ICICI-BME.2011.6108605

7. Mahalakshmi T., Muthaiah R. \& Swaminathan P. (2012). Review article: an overview of template matching technique in image processing. Research Journal of Applied Sciences, Engineering and Technology 4(24): 5469 - 5473. 
8. Meijering E.H.W., Zuiderveld K.J. \& Viergever M.A. (1999). Image registration for digital subtraction angiography. International Journal of Computer Vision 31(2): 227 - 246.

DOI: https://doi.org/10.1023/A:1008074100927

9. Moscheni F., Dufaux F. \& Kunt M. (1995). A new two-stage global/local motion estimation based on a background/ foreground segmentation. International Conference on Acoustics, Speech, and Signal Processing (ICASSP-95), volume 4, pp. $2261-2264$.

DOI: https://doi.org/10.1109/ICASSP.1995.479941

10. Shechter G., Resar J.R. \& McVeigh E.R. (2006). Displacement and velocity of the coronary arteries: cardiac and respiratory motion. IEEE Transactions on Medical Imaging 25(3): $369-375$.

DOI: https://doi.org/10.1109/TMI.2005.862752

11. Sinha Roy A., Back M.R., Khoury S.F., Schneeberger E.W., Back L.H., Velury V.V., Millard R.W. \& Banerjee R.K. (2008). Functional and anatomical diagnosis of coronary artery stenoses. Journal of Surgical Research 150(1): $24-33$.

DOI: https://doi.org/10.1016/j.jss.2007.10.018

12. Stansfield S.A. (1986). ANGY: a rule-based expert system for automatic segmentation of coronary vessels from digital subtracted angiograms. IEEE Transactions on Pattern Analysis and Machine Intelligence 8(2): 188 - 199.

DOI: https://doi.org/10.1109/TPAMI.1986.4767772

13. Suh W.M., Seto A.H., Margey R.J.P., Cruz-Gonzalez I. \& Jang I.-K. (2011). Intravascular detection of the vulnerable plaque. Circulation: Cardiovascular Imaging 4(2): 169 178.

DOI: https://doi.org/10.1161/CIRCIMAGING.110.958777

14. Takagi A., Tsurumi Y., Magosaki N., Suzuki K., Nakamura K. \& Kasanuki H. (1999). Significance of angiographic haziness at the distal stent edge: analysis by intravascular ultrasound and quantitative coronary angiography. Journal of Cardiology 33(6): 307 - 316 .

15. Tanimoto S.L. (1981). Template matching in pyramids. Computer Graphics and Image Processing 16(4): 356-369.

16. Tonino P.A.L., Fearon W.F., De Bruyne B., Oldroyd K.G., Leesar M.A., Ver Lee P.N., MacCarthy P.A., van't Veer M. \& Pijls N.H.J. (2010). Angiographic versus functional severity of coronary artery stenoses in the FAME study fractional flow reserve versus angiography in multivessel evaluation. Journal of the American College of Cardiology 55(25): 2816 - 2821 .

DOI: https://doi.org/10.1016/j.jacc.2009.11.096

17. Truc P.T.H., Khan M.A.U., Lee Y.-K., Lee S. \& Kim T.S. (2009). Vessel enhancement filter using directional filter bank. Computer Vision and Image Understanding 113(1): $101-112$.

DOI: https://doi.org/10.1016/j.cviu.2008.07.009

18. Wagner G.S., Pahlm O. \& Kjell C.N. (2011). Multimodal Cardiovascular Imaging: Principles and Clinical Applications, $1^{\text {st }}$ edition, pp. $71-80$. McGraw-Hill Medical.

19. Wong J.T., Le H., Suh W.M., Chalyan D.A., Mehraien T., Kern M.J., Kassab G.S. \& Molloi S. (2012). Quantification of fractional flow reserve based on angiographic image data. International Journal of Cardiovascular Imaging 28(1): $13-22$.

DOI: https://doi.org/10.1007/s10554-010-9767-0

20. Yamamoto M., Okura Y., Ishihara M., Kagemoto M., Harada K. \& Ishida T. (2009). Development of digital subtraction angiography for coronary artery. Journal of Digital Imaging 22(3): 319 - 325 .

DOI: https://doi.org/10.1007/s10278-008-9108-1

21. Zheng S. \& Weirong D. (2009). Analysis of coronary arterial dynamics from X-ray angiographic sequences. Second International Symposium on Computational Intelligence and Design (ISCID), 12 - 14 December, pp. $201-204$.

DOI: https://doi.org/10.1109/ISCID.2009.57 